\title{
Hypercholesterolemia induces T cell expansion in humanized immune mice
}

\author{
Jonathan D. Proto, ${ }^{1}$ Amanda C. Doran, ${ }^{1}$ Manikandan Subramanian, ${ }^{1}$ Hui Wang, ${ }^{2,3}$ Mingyou Zhang, ${ }^{2}$ Erdi Sozen, ${ }^{1,4}$ \\ Christina C. Rymond, ${ }^{1}$ George Kuriakose, ${ }^{1}$ Vivette D'Agati, ${ }^{5}$ Robert Winchester, ${ }^{1}$ Megan Sykes, $, 1,2,6$ \\ Yong-Guang Yang, ${ }^{1,2,3}$ and Ira Tabas ${ }^{1,7}$
}

\begin{abstract}
'Department of Medicine, ${ }^{2}$ Columbia Center for Translational Immunology, and ${ }^{3}$ Humanized Mouse Core, Columbia University Medical Center, New York, New York, USA. ${ }^{4}$ Department of Biochemistry, Faculty of Medicine, Genetic and Metabolic Diseases Research and Investigation Center (GEHAM), Marmara University, Istanbul, Turkey. ${ }^{5}$ Department of Pathology and Cell Biology, ${ }^{6}$ Department of Microbiology \& Immunology and Department of Surgery, and 'Department of Physiology and Cellular Biophysics, Columbia University Medical Center, New York, New York, USA
\end{abstract}

\begin{abstract}
Emerging data suggest that hypercholesterolemia has stimulatory effects on adaptive immunity and that these effects can promote atherosclerosis and perhaps other inflammatory diseases. However, research in this area has relied primarily on inbred strains of mice whose adaptive immune system can differ substantially from that of humans. Moreover, the genetically induced hypercholesterolemia in these models typically results in plasma cholesterol levels that are much higher than those in most humans. To overcome these obstacles, we studied human immune system-reconstituted mice (hu-mice) rendered hypercholesterolemic by treatment with adeno-associated virus 8-proprotein convertase subtilisin/kexin type 9 (AAV8-PCSK9) and a high-fat/high-cholesterol Western-type diet (WD). These mice had a high percentage of human T cells and moderate hypercholesterolemia. Compared with hu-mice that had lower plasma cholesterol, the PCSK9-WD mice developed a T cell-mediated inflammatory response in the lung and liver. Human CD4+ and CD8 ${ }^{+} \mathrm{T}$ cells bearing an effector memory phenotype were significantly elevated in the blood, spleen, and lungs of PCSK9-WD hu-mice, whereas splenic and circulating regulatory $T$ cells were reduced. These data show that moderately high plasma cholesterol can disrupt human T cell homeostasis in vivo. This process may not only exacerbate atherosclerosis, but also contribute to $\mathrm{T}$ cell-mediated inflammatory diseases in the hypercholesterolemia setting.
\end{abstract}

\section{Introduction}

Cardiovascular disease (CVD) remains the number one cause of death in the Western world. Dyslipidemia, the elevation of low-density lipoprotein cholesterol (LDL-C), is a critical risk factor for developing atherosclerosis, which is the underlying cause of CVD (1). In addition to traditional risk factors, accumulating evidence suggests that engagement of adaptive immunity also plays a causal role in CVD development. Increased circulating $\mathrm{T}$ effector memory (TEM) cells are present in patients with CVD and correlate with LDL-C levels (2). Indeed, several lines of evidence link $\mathrm{T}$ cell homeostasis to cholesterol metabolism (3-5). However, the majority of these studies have been carried out in mice. CVD researchers have adopted the inbred C57BL/6J mouse as the gold standard, and yet this mouse has strong $\mathrm{T}$ cell subset biases that are not reflected in the more complex human immune system $(6,7)$. Mice with a humanized immune system (hu-mice) represent a novel avenue for studying the roles of high cholesterol in human immune cell function. Several strains of mice have been optimized for human hematopoietic stem cell transplantation and functional engraftment without rejection.

Conflict of interest: The authors have declared that no conflict of interest exists. Submitted: September 29, 2017; Accepted: March 13, 2018.

Reference information: J Clin Invest. 2018;128(6):2370-2375.

https://doi.org/10.1172/JCI97785.
One particular strain, the NOD-SCID gamma (NSG) mouse, lacks T cells, B cells, and natural killer cells and has defects in innate immunity (8). Following sublethal irradiation, NSG mice can be reconstituted with human fetal thymus grafts and fetal liver $\mathrm{CD}_{3} 4^{+}$hematopoietic stem cells, resulting in excellent reconstitution with human $\mathrm{T}$ cells and $\mathrm{B}$ cells $(9,10)$. Thus, we endeavored to use the NSG hu-mouse model to study the effect of elevated cholesterol on human T cells.

In order to induce hypercholesterolemia in hu-mice, we used a technique in which a gain-of-function mutant of proprotein convertase subtilisin/kexin type 9 (PCSK9), a negative regulator of hepatic LDL receptors (LDLRs), is expressed. PCSK9 decreases LDLR levels on hepatocytes, resulting in elevated plasma LDL-C (11). Combined with a high-cholesterol/high-fat Western-type diet (WD), expression of gain-of-function PCSK9 mutant by adenoassociated virus 8-mediated (AAV8-PCSK9) gene transduction results in elevated cholesterol. Because a single AAV8-gene injection results in lifelong expression of the gene in nondividing cells without an inflammatory response, this tool enabled us to study the effects of high cholesterol over several weeks. Interestingly, this method produced a level of plasma cholesterol in hu-mice that was much more closely aligned with that in hypercholesterolemic humans, whereas most mouse models use genetic mutations to induce hypercholesterolemia (i.e., deletion of the LDL receptor or apolipoprotein E), which increases plasma cholesterol to 

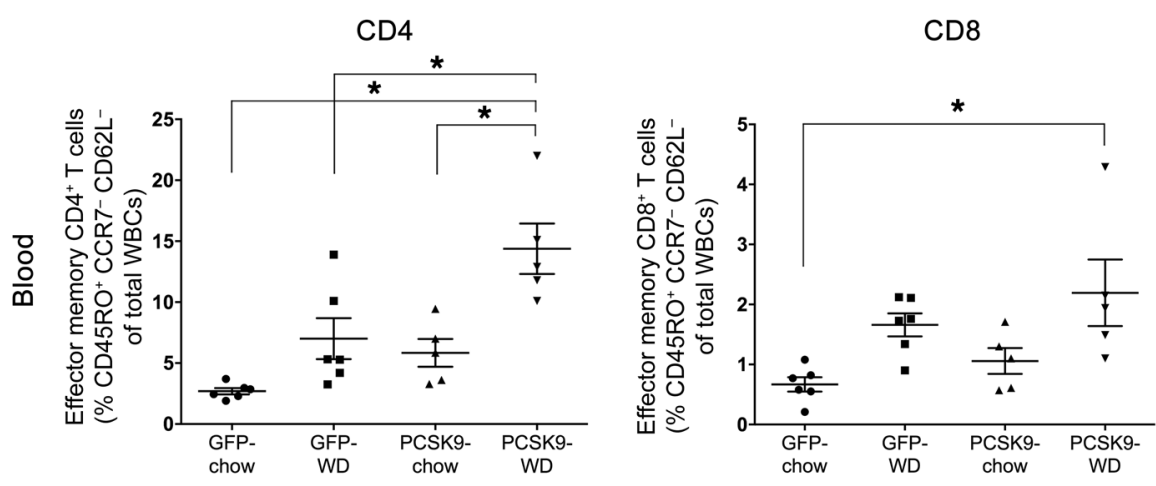

Figure 1. Human $\mathrm{CD4}^{+}$and $\mathrm{CD8} 8^{+}$effector memory $T$ cells are increased in PCSK9-WD mice. CD45RO+ CCR7- CD62L- T cells were analyzed in the blood, spleen, and lung of the indicated groups of mice using flow cytometry. Data are expressed as a percentage of total CD4+ or CD8 ${ }^{+}$ cells among total (human plus mouse) white blood cells ( $n=5-6$ mice per group; ${ }^{*} P<0.05$, 1-way ANOVA, Tukey's post hoc analysis).
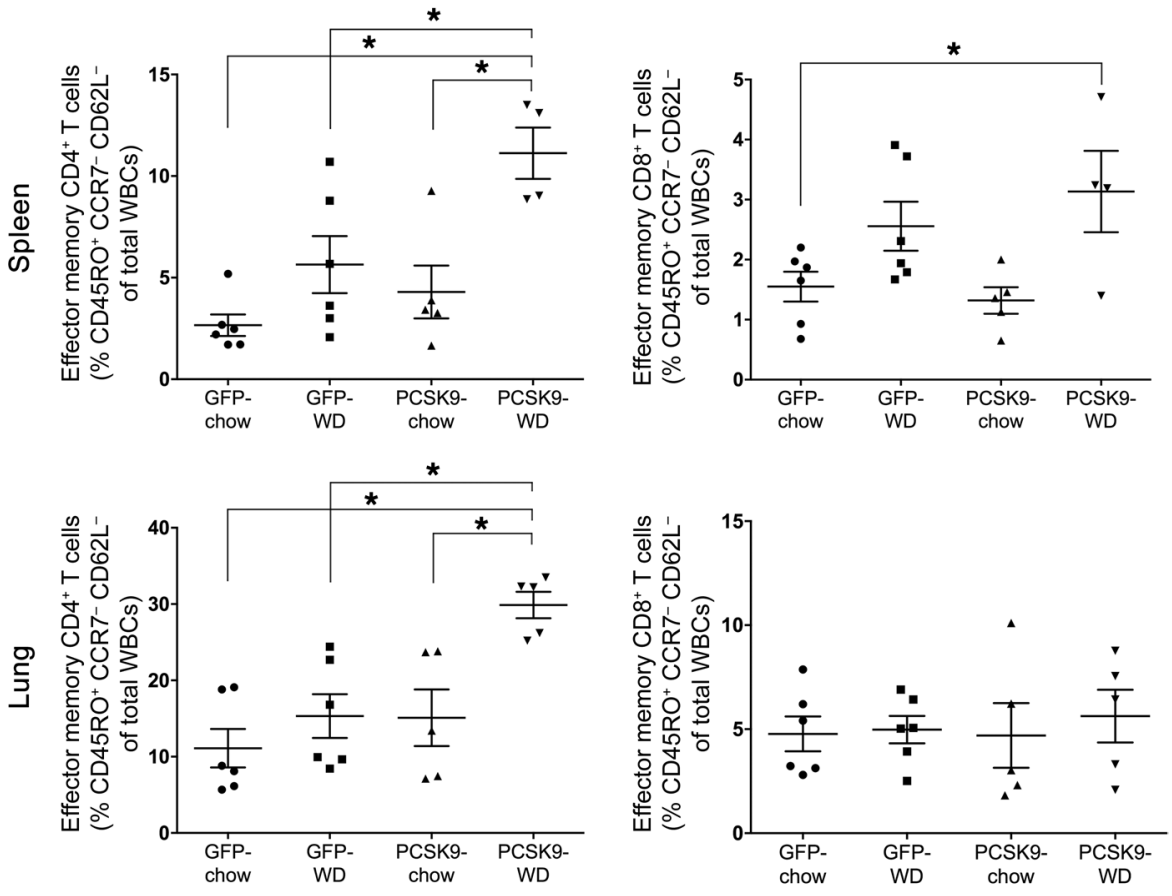

much higher levels than observed in most humans. We report here that moderately high cholesterol in hu-mice was associated with increased TEM cells and decreased regulatory $\mathrm{T}$ cells, resulting in a $\mathrm{T}$ cell autoimmune phenotype, particularly in the lung and liver. Thus, hypercholesterolemia can activate an adaptive immune response in mice with human $\mathrm{T}$ cells. This process may contribute to atherosclerosis and other inflammatory diseases associated with hypercholesterolemia.

\section{Results and Discussion}

The first cohort of mice was injected with AAV8 carrying a gain-offunction PCSK9 gene and fed a WD (PCSK9-WD) approximately 8-9 weeks after transplantation of fetal liver $\mathrm{CD}^{-} 4^{+}$cells and thymus into NSG mice (hu-mice). Control hu-mice were injected with AAV8-GFP and fed normal mouse chow (GFP-chow). Both vectors were under the control of the thyroxine binding globulin (TBG) promoter, allowing for hepatocyte-specific expression (12). In comparison with most murine models of hypercholesterolemia, in which total plasma cholesterol levels rise above $1,000 \mathrm{mg} / \mathrm{dl}$ (13), PCSK9-WD mice demonstrated a plasma cholesterol value of approximately $300 \mathrm{mg} / \mathrm{dl}$ (Supplemental Figure 1A; supplemental material available online with this article; https://doi.org/10.1172/
JCI97785DS1), which is much closer to the level seen in most humans with hypercholesterolemia ( $\geq 200 \mathrm{mg} / \mathrm{dl}$ ). Of note, this value is lower than previously published values for WD-fed C57BL/6 mice treated with AAV8 gain-of-function PCSK9 ( 850 $\mathrm{mg} / \mathrm{dl})$ (11). The lower value in our study appears to be related to irradiation, not genetic background, because WD-fed, nonirradiated NOD mice treated with AAV8 gain-of-function PCSK9 achieved a plasma cholesterol concentration of $715 \pm 73 \mathrm{mg} / \mathrm{dl}$. Unexpectedly, the PCSK9-WD mice became lethargic 10.5 weeks after AAV injection, corresponding to 19 weeks after transplant. Although human $\mathrm{CD}_{14}{ }^{+}$myeloid cell and human $\mathrm{CD}^{+} \mathrm{T}$ cell reconstitution did not differ among groups (Supplemental Figure 1, B and C), comparison of liver and lung tissue from PCSK9-WD mice with that from GFP-chow mice showed extensive mononuclear cell inflammation, and the livers showed evidence of fibrosis (Supplemental Figures 2 and 3). Immunostaining of lung tissue sections revealed that the infiltrating mononuclear cells were primarily human $\mathrm{CD}^{+} \mathrm{T}$ cells (Supplemental Figure 4).

For the second cohort of mice, we transduced hu-mice with either AAV8-TBG-eGFP or AAV8-TBG-PCSK9 combined with chow diet (GFP-chow, PCSK9-chow) or WD (GFP-WD, PCSK9WD) at 12 weeks after transplant. At 3 weeks after viral trans- 
A
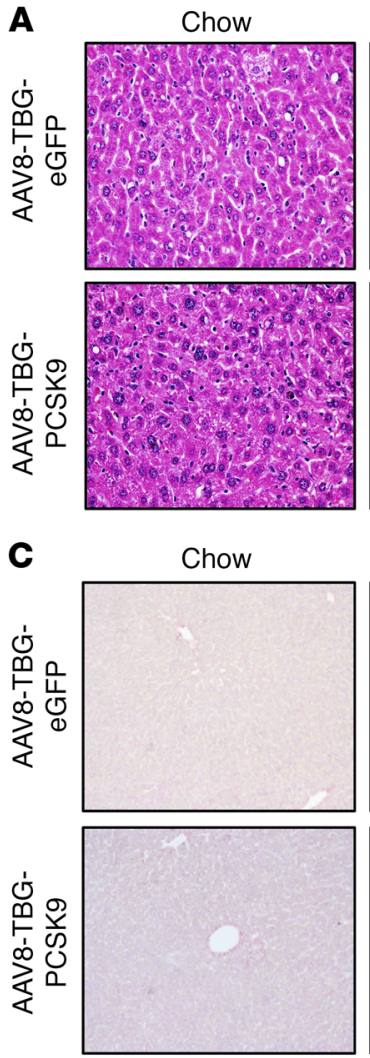

E

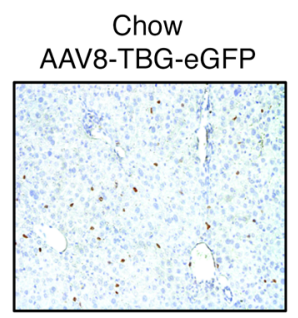

Western

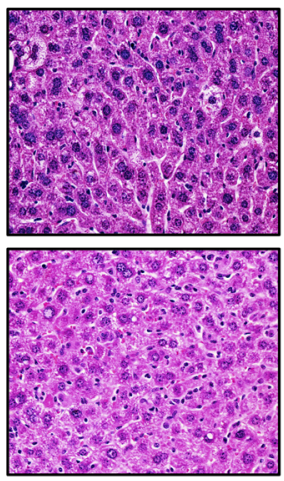

Western

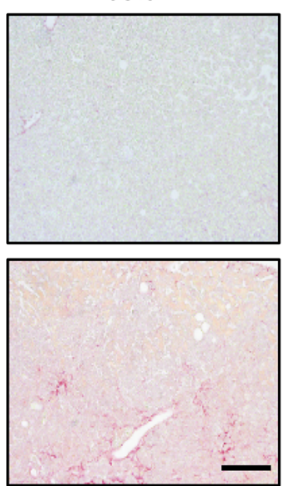

Western AAV8-TBG-PCSK9

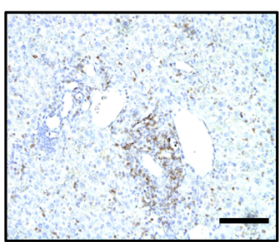

B

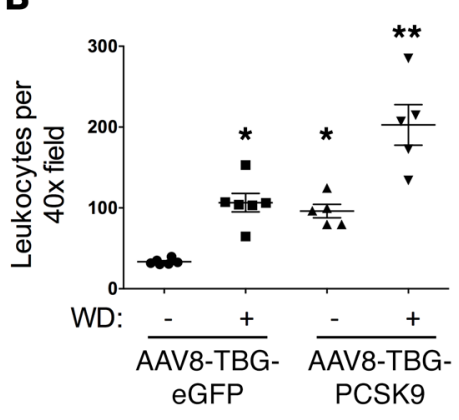

D

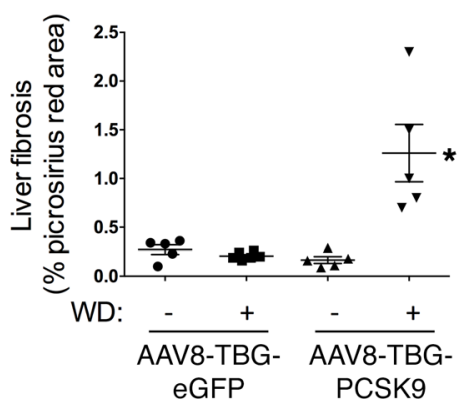

Figure 2. Liver inflammation is increased in PCSK9-WD mice. (A and B) Liver sections were stained with $\mathrm{H} \& \mathrm{E}$ and leukocytes were counted per $40 \times$ field of view (scale bar, $100 \mu \mathrm{m})(n=5-6$ mice per group; ${ }^{*} P<0.05$ vs. GFP-chow, ${ }^{* *} P<$ 0.05 vs. all other groups). (C and D) Liver sections were stained with picrosirius red to assess fibrosis (scale bar, $200 \mu \mathrm{m}$ ). Images were quantified for the percent area of fibrosis ( $n=5-6$ mice per group; ${ }^{*} P<0.05$ vs. all other groups). ( $E$ and F) Liver sections were stained with human antiCD3 and positive cells were counted per $20 \times$ field of view (scale bar, $200 \mu \mathrm{m})(n=5-6$ mice per group; ${ }^{*} P<0.05$ ). Analysis by 1 -way ANOVA and Sidak's post hoc analysis (B and $\mathbf{D}$ ) or 2-tailed Student's $t$ test $(\mathbf{F})$.
$\mathbf{F}$

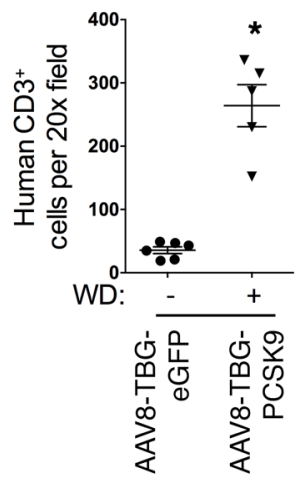

duction, PCSK9-WD mice had elevated plasma cholesterol as before, with a more moderate effect seen in PCSK9-chow mice and only a trend toward slightly higher plasma cholesterol in GFP-WD mice (Supplemental Figure 5A). Body weights among all 4 groups of mice were similar (Supplemental Figure 5B). At 6 weeks after transduction (19 weeks after transplant), the PCSK9-WD group demonstrated a significant increase in circulating human $T$ cells and a relative decrease in circulating human B cells compared with the GFP-chow group (Supplemental Figure 6). Consistent with our prior report (14), human myeloid reconstitution was low $(10-20 \%$ of total WBCs) but present at all time points assessed. At the time of sacrifice (12 weeks after transduction and 25 weeks after transplant), we assessed human $\mathrm{T}$ cell subsets in blood, spleen, and lung and found that human $\mathrm{CD}^{+}{ }^{+} \mathrm{TEM}$ cells $\left(\mathrm{CD} 45 \mathrm{RO}^{+} \mathrm{CCR}^{-} \mathrm{CD}^{-} \mathrm{L}^{-}\right)$were significantly increased in all 3 compartments in PCSK9-WD mice (Figure 1).
CD8 ${ }^{+}$TEM cells were significantly increased in both the blood and the spleen. As with the previous experiment, PCSK9-WD livers had increased leukocyte infiltration and fibrosis, and in this case intermediate phenotypes were seen with the GFPWD and PCSK9-chow cohorts (Figure 2, A-D). Immunohistochemical analysis of the livers revealed an approximately 5 -fold increase in human $\mathrm{CD}^{+} \mathrm{T}$ cells in the livers of PCKS9-WD mice compared with GFP-chow mice (Figure 2, E and F). Similarly, histological analysis of lung tissue revealed perivascular and peribronchial inflammation (Figure 3, A and B). Flow analysis of lung human $\mathrm{CD} 45^{+}$cells showed an increased percentage of human T cells in the PCSK9-WD group (Figure 3C). In this cohort, we also examined the kidney for evidence of inflammation. H\&E-stained sections were reviewed by a renal pathologist blinded to the groupings and scored for interstitial, perivascular, subcapsular, and peripelvic inflammation and for interstitial 
A
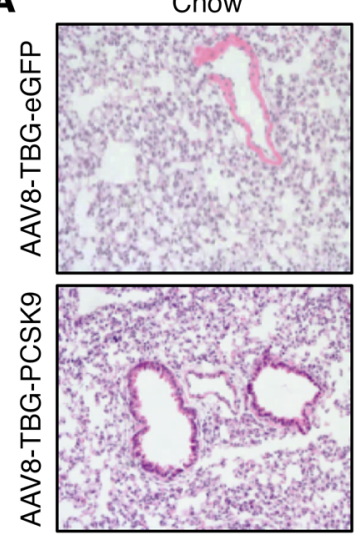

Western
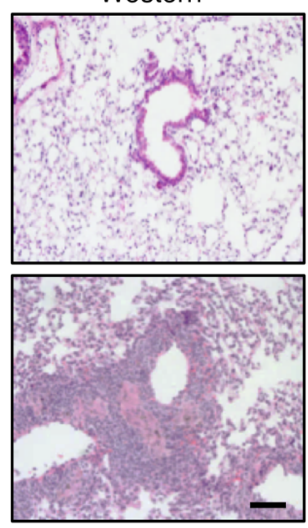

B

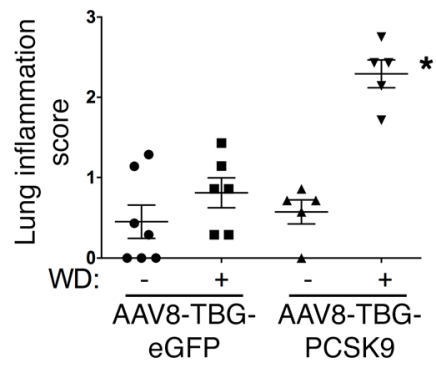

C

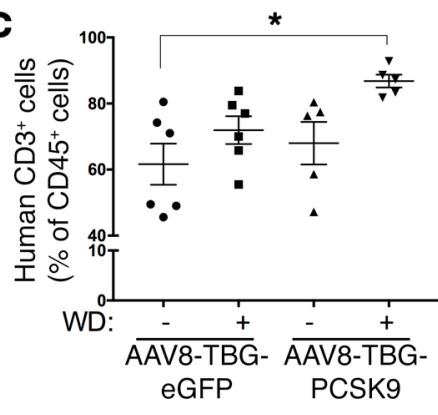

Figure 3. Lung inflammation is increased in PCSIK9-WD mice. (A and B) Lung sections were stained with H\&E (scale bar, $200 \mu \mathrm{m}$ ) and scored for perivascular and peribronchial inflammation ( $n=5-6$ mice per group; ${ }^{*} P<0.05$ vs. all other groups by 1-way ANOVA and Sidak's post hoc analysis). (C) Analysis of human $\mathrm{CD}^{2} 5^{+}$and $\mathrm{CD}^{+}$cells by flow cytometry ( $n=5-6$ mice per group; ${ }^{*} P<0.05$ vs. GFP-chow by Kruskal-Wallis test with Dunn's multiple comparisons test).

fibrosis. The percentage of inflammation was determined for each anatomic site, based on an estimated percentage of total cortical area and with inflammation in each cross-section examined (Supplemental Table 1 and Supplemental Figure 7). While not as striking as the findings in the liver and lung, there was an overall trend toward increased inflammation and fibrosis in the PCSK9-WD group compared with the other groups. Further, we analyzed pancreatic sections from GFP-chow and PCSK9-WD mice and found significant $\mathrm{CD}^{+} \mathrm{T}$ cell perivascular infiltrates in the latter group (Supplemental Figure 8). Given the relationship among hypercholesterolemia, TEM cells, and atherosclerosis, we asked whether any aortic lesion development occurred in these mice. At the time of sacrifice (12 weeks after transduction, 25 weeks after transplant), aortic roots were harvested and assessed for presence of atherosclerosis. No significant lesions were found in any groups (Supplemental Figure 9). This finding was not unexpected, as hypercholesterolemia was only moderate and NOD mice have previously been reported to be resistant to diet-induced hypercholesterolemia and atherosclerosis compared with C57BL/6 mice, which is the strain typically used for atherosclerosis studies (15).

In association with the increase in TEM cells, the proportion of human Tregs $\left(\mathrm{CD} 4^{+} \mathrm{CD} 127^{\text {low }} \mathrm{CD} 25^{+}\right.$Foxp $\left.^{+}\right)$was decreased in the blood and spleen of PCSK9-WD mice (Figure 4). CCR5 ${ }^{+} \mathrm{T}$ cells, which have been implicated in autoimmune diseases such as rheumatoid arthritis and multiple sclerosis $(16,17)$, were elevated in both $\mathrm{CD}^{+}$and $\mathrm{CD}^{+}$subsets in the spleens of PCSK9-WD mice (Figure 4, B and C). However, anti-nuclear antibodies were not detected in any of the 4 cohorts (Supplemental Figure 10).

Memory $\mathrm{T}$ cell population expansion has previously been reported for murine models of dyslipidemia and atherosclerosis $(18,19)$. However, to our knowledge, a longitudinal study of effector memory $\mathrm{T}$ cell dynamics in hypercholesterolemic mice has not been previously reported. Thus, we compared $\mathrm{T}$ cell subsets in the spleens of chow-fed WT and WD-fed $\mathrm{Ldlr}^{--}$mice at 2, 12, and 15 weeks. After 15 weeks of diet, splenic $\mathrm{CD}^{+}{ }^{+}$and $\mathrm{CD} 8^{+} \mathrm{TEM}$ cells (CD44 ${ }^{+}$CD62L $\left.\mathrm{L}^{\text {low/neg }}\right)$ were significantly increased in WD-fed $\mathrm{Ldlr}^{-/}$ mice compared with age-matched chow-fed WT mice (Supple- mental Figure 11, A and B). Further, the ratio of Treg/CD4+ TEM cells, which has been shown to be predictive of atherosclerosis in hypercholesterolemic models (20), was also significantly decreased after 15 weeks (Supplemental Figure 11C). To further verify the link between high cholesterol and $\mathrm{T}$ cell effector memory expansion, we compared PCSK9-WD and PCSK9-chow mice. The PCSK9-WD mice, but not the PCSK9-chow mice, showed an increase in CD4 ${ }^{+}$ and $\mathrm{CD}^{+}$TEM cells in the spleen after 10 weeks (Supplemental Figure 11, D and E). The increase in TEM cells appeared to correlate positively with cholesterol levels (Supplemental Table 2). These data support the conclusion that plasma cholesterol levels affect murine and human TEM cell expansion in mouse and hu-mouse models of hypercholesterolemia, respectively.

TEM and central memory $\mathrm{T}$ cells are able to rapidly respond to antigen reexposure and carry out their effector functions. TEM cells have been associated with aging and autoimmune diseases in humans. For example, one study reported that $30-40 \%$ of $\mathrm{CD}^{+}$ TEM cells demonstrate a cytokine-responsive phenotype in lymph nodes, spleen, and blood as humans age, suggesting that TEM cells can potentially be expanded in a non-TCR-specific manner (21). Among the autoimmune diseases driven by TEM cells are type 1 diabetes, rheumatoid arthritis, and multiple sclerosis (22). In this investigation, we have identified a putative autoimmune pathology in humanized NSG mice with moderately high cholesterol, characterized by increased $\mathrm{CD}^{+}$and $\mathrm{CD} 8^{+}$TEM cell subsets, decreased Tregs, and multiorgan pathology, including inflammation and fibrosis. Hypercholesterolemia-induced expansion of TEM cells is of particular interest given the association between TEM cells and atherosclerosis in animal models and in humans (2). While the most robust effect was observed in TEM cells in PCSK9-WD mice, there was also a mild, non-statistically significant expansion of splenic $\mathrm{CD}^{+}{ }^{+} \mathrm{TEM}$ cells seen in hu-mice on Western diet alone (GFP-WD) or receiving AAV8-TBG-PCSK9 alone (PCSK9-chow). Concomitantly, we observed a modest increase in leukocytes in the livers of GFP-WD mice or PCSK9-chow mice, although these mice did not have increased liver fibrosis. This suggests that even modest elevations in plasma cholesterol may be able to stimulate the differentiation of TEM cells. While cholesterol would seem to be the common 
A

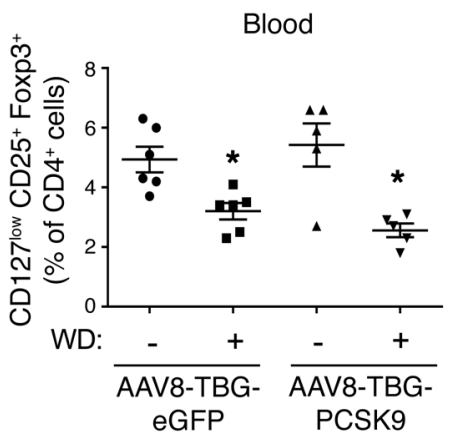

B

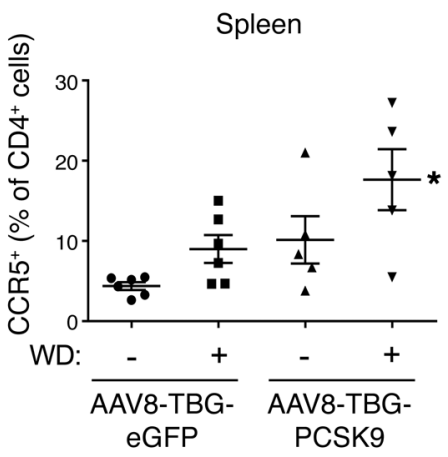

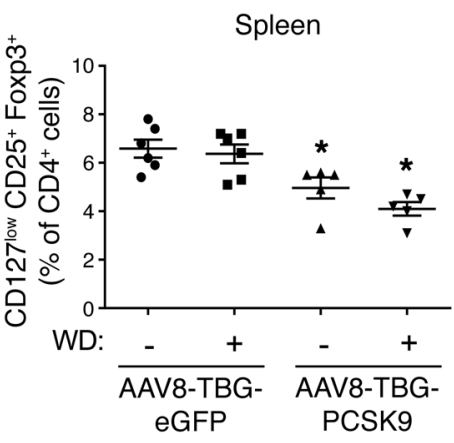

C

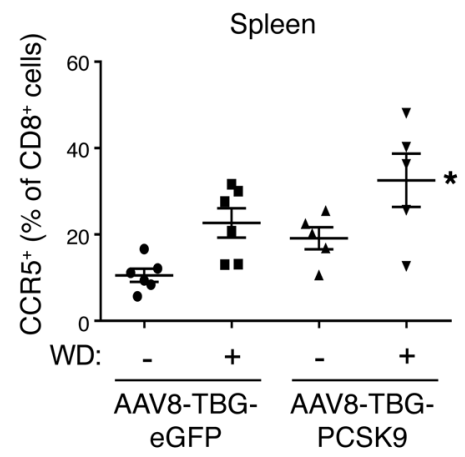

Figure 4. Human regulatory $T$ cells are reduced and CCR5 T cells are increased in PCSK9-WD mice. (A) Human CD4 ${ }^{+}$ CD127 low $\mathrm{CD}^{2} 5^{+}$Foxp $^{+}$cells were analyzed in the blood and spleen of the indicated groups of mice using flow cytometry ( $n=5-6$ mice per group; ${ }^{*} P<0.05$ vs. GFP-chow, 1-way ANOVA, Tukey's post hoc analysis). (B and C) Human CCR5 $5^{+}$CD4 ${ }^{+}$ and CCR5 ${ }^{+} \mathrm{CD}^{+} \mathrm{T}$ cells in the spleen were analyzed by flow cytometry ( $n=5-6$ mice per group; ${ }^{*} P<0.05$ vs. GFP-chow, 1-way ANOVA, Tukey's post hoc analysis). factor driving these changes, we cannot rule out the contribution of other dietary elements such as saturated fat in the Western diet, which may have independent or additive effects to cholesterol itself.

In line with our findings, hyperlipidemia in mice has been reported to accelerate cardiac allograft rejection via a $\mathrm{T}$ cellmediated process (23). Consistent with this report, we found increased $\mathrm{CD}^{+}$and $\mathrm{CD}^{+} \mathrm{TEM}$ cells in hypercholesterolemic $\mathrm{Ldlr}^{-1-}$ mice and AAV8-PCSK9-transduced WT mice after WD feeding. Future investigations will be necessary to determine the mechanism linking hypercholesterolemia to TEM cell expansion, but current evidence in the literature raises the possibility that enrichment of plasma membrane cholesterol in $\mathrm{T}$ cells may be involved. For example, increased membrane cholesterol has been reported to promote Th1 differentiation of $\mathrm{CD} 4^{+} \mathrm{T}$ cells (24). Cholesterol in the plasma membrane has been shown to directly bind the TCR and mediate nanoclustering, which enhances the avidity of TCR interaction with antigen $(4,25)$. Interestingly, there are reports that $\mathrm{T}$ cells from patients with systemic lupus erythematosus (SLE) have elevated levels of cholesterol and disrupted localization of the raft-associated signaling molecule Lck $(26,27)$. Treatment of SLE T cells with a statin decreased cholesterol, normalized Lck localization, and reduced production of the cytokines IL-10 and IL-6 (26).

In contrast to murine models of hypercholesterolemia, in which values reach over $1,000 \mathrm{mg} / \mathrm{dl}$, the use of humanized immune mice and AAV8-TBG-PCSK9 has allowed us to study the human immune system in the setting of cholesterol levels that are closer to those seen in hypercholesterolemic humans. Moderate hypercholesterolemia induces atherosclerosis in mice only after many months, and the mice in this study became ill with inflammatory liver and lung disease and eventually were sacrificed before lesions could develop. However, as demonstrated here, these mice can be used to study the pathophysiology of shorter-term disease and predisease processes in which dyslipidemia contributes to $\mathrm{T}$ cell autoimmunity. Moreover, future modifications of the model may make it possible to study longer term disease processes like atherosclerosis. In all cases, the use of humanized mice may provide a valuable model to conceive and validate therapeutic strategies prior to actual testing in humans.

\section{Methods}

For additional methods, please see supplemental material.

Mice. C57BL/6J (WT), B6.129S7-LdlrtmlHer $/ \mathrm{J}$ ( $\left.L d l r^{-/}\right)$, NOD.CgPrkdc scid-Tg(IL3)1YgyTg(CSF2)2YgyTg(KITLG)3Ygy (NOD.scid porcine IL-3-CSF2-KITLG), and NOD.Cg-Prkdc scid Il2rg ${ }^{\text {tmlwj }} / / S z J$ (NODscid IL2r $\gamma^{\text {null }}$, abbreviated as NSG) mice were purchased from Jackson Laboratories (stock numbers 000664, 002207, 005557, and 004644, respectively) and housed in a specific pathogen-free microisolator environment. To generate humanized mice, NOD.scid porcine IL-3CSF2-KITLG mice (cohort 1) or NSG mice (cohort 2) were conditioned with sublethal (1.0 Gy) total-body irradiation. Human fetal thymus fragments $\left(1 \mathrm{~mm}^{3}\right)$ were implanted under the kidney capsule followed by intravenous injection with $2 \times 10^{5}$ autologous human fetal CD34 ${ }^{+}$ cells within 24 hours of fetal thymus transplantation. All recipients were injected intraperitoneally with anti-human CD2 monoclonal antibody (BTI 322; $400 \mu \mathrm{g} / \mathrm{mouse}$ ) on days 0 and 7. Additional details are included in the supplemental methods.

Statistics. All results are mean \pm SEM. Normality was determined using D’Agostino-Pearson and/or Shapiro-Wilk normality testing. $P$ values for normally distributed data were calculated using either a 2-tailed Student's $t$ test or 1-way ANOVA with either Tukey's or Sidak's post hoc analysis as indicated in the figure legends. $P$ values for nonnormally distributed data were calculated using the Mann-Whitney rank sum test or the Kruskal-Wallis test with Dunn's multiple comparisons testing. A $P<0.05$ was considered significant. Pearson's correlation coefficients $\left(r^{2}\right)$ are represented by $\mathrm{R}^{2}$ in Supplemental Table 2. 
Study approval. All animal protocols were approved by the Columbia University IACUC.

\section{Author contributions}

JDP, RW, M Sykes, YGY, and IT conceived and designed the research. JDP, ACD, M Subramanian, HW, MZ, ES, CCR, GK, and YGY conducted experiments. VD conducted the pathological analysis of the kidneys from the hu-mice. All authors contributed to data analysis. JDP, ACD, and IT wrote the manuscript, and all authors contributed to manuscript editing.

\section{Acknowledgments}

This work was supported by NIH T32 training grant HL00734328 (to JDP); an American Heart Association Fellow-to-Faculty award (to ACD); NIH grants DK106436 and AR064473 (to RW), AI064569 (to YGY), UC4DK104207, R01DK103585, and P01AI045897 (to M Sykes), and HL132412, HL075662, and HL127464 (to IT); and by an Interdisciplinary Research Initia- tives Seed (IRIS) Fund Program award from Columbia University Medical Center (to IT and M Sykes). These studies used flow cytometry facilities of the Columbia Center for Translational Immunology Flow Cytometry Core, the Columbia Diabetes and Endocrinology Research Center, and the Columbia Cancer Center, funded, respectively, through an NIH Shared Instrumentation Grant 1S10RR027050 and NIH Center Grants 5P30DK063608 and P30CA013696.

Address correspondence to: Ira Tabas, Department of Medicine, Columbia University Medical Center, 630 W 168th Street, New York, New York 10032, USA. Phone: 212.305.5669; Email: iat1@ cumc.columbia.edu.

JDP's present address is: Sanofi, Framingham, Massachusetts, USA.

M. Subramanian's present address is: CSIR-Institute of Genomics and Integrative Biology, New Delhi, India.
1. Yusuf S, et al. Effect of potentially modifiable risk factors associated with myocardial infarction in 52 countries (the INTERHEART study): case-control study. Lancet. 2004;364(9438):937-952.

2. Ammirati E, et al. Effector memory T cells are associated with atherosclerosis in humans and animal models. JAm Heart Assoc. 2012;1(1):27-41.

3. Bensinger SJ, et al. LXR signaling couples sterol metabolism to proliferation in the acquired immune response. Cell. 2008;134(1):97-111.

4. Yang $W$, et al. Potentiating the antitumour response of CD8(+) T cells by modulating cholesterol metabolism. Nature. 2016;531(7596):651-655.

5. Armstrong AJ, Gebre AK, Parks JS, Hedrick CC. ATP-binding cassette transporter G1 negatively regulates thymocyte and peripheral lymphocyte proliferation. JImmunol. 2010;184(1):173-183.

6. Schulte S, Sukhova GK, Libby P. Genetically programmed biases in Th1 and Th2 immune responses modulate atherogenesis. Am J Pathol. 2008;172(6):1500-1508.

7. Wakeham J, Wang J, Xing Z. Genetically determined disparate innate and adaptive cell-mediated immune responses to pulmonary mycobacterium bovis BCG infection in C57BL/6 and BALB/c mice. Infect Immun. 2000;68(12):6946-6953.

8. Shultz LD, et al. Human lymphoid and myeloid cell development in NOD/LtSz-scid IL2R gamma null mice engrafted with mobilized human hemopoietic stem cells. JImmunol. 2005;174(10):6477-6489.

9. Lan P, Tonomura N, Shimizu A, Wang S, Yang YG. Reconstitution of a functional human immune system in immunodeficient mice through combined human fetal thymus/liver and CD34+ cell transplantation. Blood. 2006;108(2):487-492.

10. Tonomura N, Habiro K, Shimizu A, Sykes M,
Yang YG. Antigen-specific human T-cell responses and T cell-dependent production of human antibodies in a humanized mouse model. Blood. 2008;111(8):4293-4296.

11. Bjørklund MM, et al. Induction of atherosclerosis in mice and hamsters without germline genetic engineering. Circ Res. 2014;114(11):1684-1689.

12. $\mathrm{Mu} \mathrm{X}$, et al. Hepatocellular carcinoma originates from hepatocytes and not from the progenitor/biliary compartment. J Clin Invest. 2015;125(10):3891-3903.

13. Ishibashi S, Brown MS, Goldstein JL, Gerard RD, Hammer RE, Herz J. Hypercholesterolemia in low density lipoprotein receptor knockout mice and its reversal by adenovirus-mediated gene delivery. J Clin Invest. 1993;92(2):883-893.

14. Tonomura N, et al. Pig islet xenograft rejection in a mouse model with an established human immune system. Xenotransplantation. 2008;15(2):129-135.

15. Keren P, George J, Keren G, Harats D. Non-obese diabetic (NOD) mice exhibit an increased cellular immune response to glycated-LDL but are resistant to high fat diet induced atherosclerosis. Atherosclerosis. 2001;157(2):285-292.

16. Qin S, et al. The chemokine receptors CXCR3 and CCR 5 mark subsets of $\mathrm{T}$ cells associated with certain inflammatory reactions. J Clin Invest. 1998;101(4):746-754.

17. Balashov KE, Rottman JB, Weiner HL, Hancock WW. CCR5(+) and CXCR3(+) T cells are increased in multiple sclerosis and their ligands MIP-1alpha and IP-10 are expressed in demyelinating brain lesions. Proc Natl Acad Sci U S A. 1999;96(12):6873-6878.

18. Ammirati E, Moroni F, Magnoni M, Camici PG. The role of $\mathrm{T}$ and $\mathrm{B}$ cells in human atherosclerosis and atherothrombosis. Clin Exp Immunol.
2015;179(2):173-187.

19. Rao LN, Ponnusamy T, Philip S, Mukhopadhyay R, Kakkar VV, Mundkur L. Hypercholesterolemia induced immune response and inflammation on progression of atherosclerosis in Apob(tm2Sgy) Ldlr(tm1Her)/J mice. Lipids. 2015;50(8):785-797.

20. Tabas I, Lichtman AH. Monocyte-macrophages and $\mathrm{T}$ cells in atherosclerosis. Immunity. 2017;47(4):621-634.

21. Thome JJ, et al. Spatial map of human T cell compartmentalization and maintenance over decades of life. Cell. 2014;159(4):814-828.

22. Devarajan P, Chen Z. Autoimmune effector memory T cells: the bad and the good. Immunol Res. 2013;57(1-3):12-22

23. Yuan J, Bagley J, Iacomini J. Hyperlipidemia promotes anti-donor Th17 responses that accelerate allograft rejection. Am J Transplant. 2015;15(9):2336-2345.

24. Surls J, Nazarov-Stoica C, Kehl M, Olsen C, Casares $\mathrm{S}$, Brumeanu TD. Increased membrane cholesterol in lymphocytes diverts T-cells toward an inflammatory response. PLoS ONE. 2012;7(6):e38733.

25. Molnár E, et al. Cholesterol and sphingomyelin drive ligand-independent T-cell antigen receptor nanoclustering. J Biol Chem. 2012;287(51):42664-42674.

26. Jury EC, Isenberg DA, Mauri C, Ehrenstein MR. Atorvastatin restores Lck expression and lipid raft-associated signaling in $\mathrm{T}$ cells from patients with systemic lupus erythematosus. J Immunol. 2006;177(10):7416-7422

27. Jury EC, Kabouridis PS, Flores-Borja F, Mageed RA, Isenberg DA. Altered lipid raft-associated signaling and ganglioside expression in T lymphocytes from patients with systemic lupus erythematosus. J Clin Invest. 2004;113(8):1176-1187. 